\title{
Bienes comunes, consenvación ambiental y economía campesina. Un estudio de caso en el Parque Nacional Cofre de Perote, Veracnuz, México
}

\author{
Janett Vallejo Román \\ Centro de Investigaciones y Estudios Superiores en Antropología Social, Xalapa, México. \\ Email: jvallejo@c iesas.edu.mx \\ J uan Carlos Rodríguez Toment \\ Universidad de Valparaíso, CINVIT, Valparaíso, Chile. \\ Email: juancarlosrodriguezt@yahoo.com
}

Recibido: 30.06 .20 | Aceptado: 13.08 .20

\begin{abstract}
Resumen: En México, el decreto de Áreas Natura les Protegidasconstituye una de lasprincipa les inic iativasgubema menta lesen tomo a la conservación a mbiental, a sumiénd ola como una de las herramientas más efec tivas para la conservación de los ecosistemas, frenar la expansión de la frontera agríc ola y otorgaridentidad a luga resseleccionados. Rec onociendo que cada área de conservación tiene particularidades a mbientales, económicas, sociales e históricas, el objetivo de este artíc ulo es discutir las tensiones que se produce entre el rango de «bien común»sujeto a la nomativa de Área Nacional Protegida y poblaciones locales que hacen uso de la naturaleza. Para ello, tomamoscomo estudio de caso el Parque Nacional Cofre de Perote, Vera cruz, México, a nalizando etnográ fic a mente las contraposic iones disc ursivas y prá c tic a sentre quienes habitan y quienes designan, pla nean, organizan y toman el control de las ANP, y las posibilida des de hacer uso sostenible de recursos. Palabras clave: Naturaleza; bienes comunes; conservación; economía campesina; área natural protegida
\end{abstract}

\section{Common goods, environmental conservation and peasant economy. A case study in Cofre de Perote National Park, Verac nuz, Mexico}

\begin{abstract}
In Mexico, the Natural Protected Areas decree is one of the main govemment initia tives regarding environmental conservation, assuming it as one of the most effective tools for the conservation of ecosystems, slowing down the expansion of the agricultural frontier and giving identity to selected sites. Recognizing that each conservation area has environmental, economic, social, and historic al particularities, the objective of this artic le is to discuss the tensions that a rise between the rank of «common good» subject to National Protected Area regulations and local populations that make use of nature. To do so, we take as a case study the Cofre de Perote National Park, Veracruz, Mexic o, ethnographically analyzing the discursive and practical oppositions between those who inhabit and those who designate, plan, organize and take control of the NPAs, and the possibilities of making sustainable use of resources.
\end{abstract}

Key words: Nature; common goods; conservation; peasant economy; protected natural area 
Bens comuns, conservação ambiental e economia camponesa. Um estudo de caso no Parque Nacional Cofre de Perote, Veracruz, México

Resumo: O decreto das Áreas Natura is Protegidas no México compõe uma das principa is inic iativas govemamenta is de conservação ambiental, assumindo-se como uma das ferramentas ma is efic azes para a conserva ção dos ec ossistemas, reta rdando a expansão da fronteira a gríc ola e conferindo uma identidade a osloca is seleciona dos. Reconhec endo que cada área de conservação possui peculiaridades ambienta is, econômicas, socia is e históric as, o objetivo deste a rtigo é disc utira stensões que suc edem entre a classific ação de «bem comum» sujeita às regulamentações de Área Nacional Protegida e às populações locais que fazem uso da natureza. Para isso, tomamos como estudo de caso o Parque Nacional Cofre de Perote, Veracruz, México, a nalisando etnograficamente os contra stes disc ursivos e práticos entre quem mora e quem institui, planeja, orga niza e assume o controle das ANP, assim como a possibilidades de fazer um uso sustentável dos recursos.

Palavras-chave: Natureza; bens comuns; conservação; ec onomia camponesa; área natural protegida.

\section{Como citar este artículo:}

Vallejo Román,J , y Rodríguez Torrent, JC. (2020). Bienesc omunes, conserva ción a mbiental y ec onomía campesina. Un estud io de caso en el Pa rque Na c ional C ofre de Perote, Vera cruz, México . Polis Revista La tinoa meric ana, (57), 115-137. doi: http://dx.doi.org/10.32735/S07186568/2020-N57-1567

\section{Introducción}

\section{La conservación de áreas protegidas}

Losońgenes de los parquesna cionales (o áreasprotegidas) poseen una connotación original pragmática que implicó, casi en todos los casos, expulsara las poblaciones residentes y desplaza rlasa ctivida destra diciona les (G uha, 1997) para desa molla rotrosfines. La erra dicación y los decretos de expropiación fueron elementos comunes para hacer valer lo que podría seractua Imente el «derecho a mbiental». El a ctor clave para ejec utar las noma sfue el Estado como propietario, de manera que lasáreasnaturales protegidas se constituyeron como una forma particular de marcar presencia institucional, definiendo, delimitando y visua liza nd o estos territorios como espec ia les (Ferrero, Arizpe y Gómez, 2013, p. 85). Desde esta perspectiva, los bienes natura les sujetos a conserva ción y puestos como valor común para fines no tradicionales (i.e. turismo, recreación y científicos), implican tanto una selección de lugares como la posibilidad de uso y consumo de «os dones de la naturaleza» (Guha, 1994, p. 139), colocando intereses de «otros» por sobre el tiempo y la tradición de poblaciones muchas vec es empobrecidasque viven en su área, dentro o fuera de la reserva (Guha, 1997). También, signific a un proceso de gobemanza a mbiental en cua nto deben ser administrados, generando prácticas reguladoras, procesos, mecanismos e incidencia de actores que afectan e influyen en el curso de las acciones y los resultados en tomo a cuestiones temitoria les y a mbientales (Ba rkin y Lemus, 2015; Baud, De Castro y Hogenboom, 2011; Faninós Dasí, 2008; Lemos y Agrawal, 2006; Rosales Ortega y Brenner, 2015); ya sea privatizándolos, regulándolos a través del Estado, o delegando su responsabilidad en poblaciones locales. Ello, con efectos «expropiatorios» y configuradores de «desa raigo» entre 
loslocales(Guha, 1997, p. 36), frente a lo que pudiese lla marse un objetivo superior: se están a gotando luga res de a lto valor ecosistémico, o están severa mente a mena za dos conforme a lenguajes de valoración (Martínez Alier, 2011).

Así, muc has de las a c titudes conserva cio nistas ac tua les deben ser entendidas como «un síntoma de la mercantilización de la naturaleza» y como «la respuesta emocional a las consec uencia sde esta merc a ntiliza ción» (Comas-d'Argemir, 1998, p. 121). Pero, sobre to do, la manifestación de una dimensión de la vida que se está perdiendo y que es necesario contener, por lo que a lgunas de las tempranas motiva ciones tenían un objetivo más político-económico, social, o incluso moral, que ambiental. Por ejemplo, el uso racional y eficiente de la madera implicaba la generación de planes y proyectos, restricciones y leyes punitivas para quienes hic ieran un «uso» diferente al establecido por las tempranas élites conservacionistas, que establecían dicha nomatividad sobre argumentos filosófic os, morales y científic os (Guha, 1997). Es decir, el uso racional y efic iente del recurso fue dicta do y nomado hegemónic a mente por un grupo que decidió las formas y prácticas permisibles de acuerdo a sus necesidadese intereses diversos, a sí como la detemina ción de los castigos para quién rompiera dicha noma, lo que más tarde se convertińa en una idea de exportación norteamericana (Guha, 1997).

Lo a nterior, signific a conceptua Imente que los recursos dentro de un área protegida alcanzan un valor híbrido, o que son valorados de manera distinta en términos de Martínez Alier (2011). Ta mb ién que los instrumentos regula to riosson igua Imente singula res, en cua nto c rean un lugar nuevo y un nicho de mercado diferente y específico, eficiente y propicio para la a cumula ción de capital en áreas donde los mercados tienen dific ulta des para ingresar y poner valor monetario. De este modo, la mercantilización de la naturaleza frente a recursosúnicoscomo losque proporcionaban los grown forest, requiere de una institucionalidad que fije las coordenadas de su apropiación. Por tanto, el Estado y la legislación crean instancias para que un área considerada valiosa pueda ser apropiada para resguardo monetario, simbólico y trascendental. De ahí que la pregunta central de este documento sea ¿cuáles son lastensiones que se produce entre el rango de «bien común»sujeto a la nomativa de Área Nacional Protegida (ANP) y poblacioneslocales que hacen uso de los recursos natura les a su alc ance?

A partir de esta breve introduc ción, el trabajo se divide en cinco a parta dos. En el primero se enuncia y expone el método de trabajo y de recolección de información. En el segundo se establece el marco de a ná lisis de áreas protegidas para introducir el ca so de estudio. En el tercero se disc uten lasideascentra les so bre losPa rquesNa ciona lesy áreas de conserva ción en Méxic o. En el cuarto, se c ara cteriza el Parque Nacional C ofre de Perote, donde exponen reflexionesen la perspec tiva del reconoc imiento y la integración de laspoblacionaleslocales. Fina Imente, en quinto se muestran resulta dos y se establecen a lgunas consideraciones finales. 


\section{Metodología}

La metodología empleada privilegia la etnografía y el enfoque relacional para mostrar las interacciones entre la sociedad y los marcos regulatorios del Estado, particularmente la manera en que se cumplen o no lasnomas en tomo al acceso y uso de los recursos en las áreas natura lesprotegidas (ANP), a sí c omo losreclamose inconformida des propiasfrente a tales restric ciones. El trabajo de campo se llevó a cabo de 2017 a 2019, en las localidades del municipio de Perote, que están ubic adas parcialmente o en su totalidad en el teritorio del Parque Nacional C ofre de Perote, Veracruz, identifica do con el nombre homónimo de la octava montaña más alta de México, 4.282 msnm. Estas comunidades son: Ejido Agua de los Pescados, El Conejo, Rancho Nuevo y El Escobillo. La información se obtuvo a través de 35 entrevista ssemiestruc tura dasa funciona rios federa les, estata lesy munic ipa les de á reas centrales como la Comisión Nacional Forestal (CONAFOR), la Comisión Nacional de Áreas Natura les Protegidas (CONANP) la Secretaria de Medio Ambiente (SEDEMA-Veracruz), y a informa ntes c laves en la s loc a lida des del ANP, como son las a utorida des ejida les, los a gentes munic ipa lese integrantes de grupos de mujeres productoras. Del total de los entrevistados 9 fueron funcionarios de alto nivel de las dependencias señala das, todos va rones con estudios de profesionales. El resto de las entrevistas (28) fueron a habitantes de los ejidos, con las siguientes ca rac terístic as: el $67 \%$ fueron hombres (19) y $33 \%$ mujeres (9) de lascua les todas participan activamente en la comunidad (8 participan en un proyecto productivo y una es representante esc olar). De los va rones, 12 oc upan distintos cargos en las estructura ejidal (presidente, tesorero, secretario o voc al); 3 son a gentes munic ipales; $y, 4$ son productores de papa, sin cargo como representante, a unque si partic ipa n en lasasa mbleasejidales. A tra vés de estas entrevistas, se buscó observar la proyec c ión de valores presentes en ca da uno de los actores sobre la figura del parque, ya que la montaña Cofré de Perote permite conduc ir agua para irriga ción agríc ola y consumo humano, que incluyen ciudadesimportantescomo Xa la pa de Enńquez (489.619 habs.). Y, que asocia da a un bosque que se ubic a en entre los 3.200 y 3.400 msnm., que produce humedad y lluvias, permite esta blecery disc utir propuestas polític as-ambientales, sensibilidades, necesidades y requerimientos diversos ante procesos desforesta dores.

Es importante mencionar que, a poco más de ocho décadas de su decreto como área protegida, fue hasta el 2015 que se concretó el Programa de Manejo, por lo que lo descrito se trata de un proceso en construcción. En este sentido, consideramos que es un ejemplo interesa nte por lo extra polable, pues permite trazar históric a mente las distintas nociones y práctic as de conserva ción a mbienta l en México, a sí c omo la rea liza ción de a bordajes críticosfrente a una figura creciente.

\section{Áreas Nacionales Protegidas}

En la lógica occidental modema, el desarrollo económico y la figura lineal del progreso se ha basa do en el control y explota ción de recursos que se a precian ima ginariamente como infinitos, lo que ha generado un proceso de «economización de la naturaleza», que incluye 
tanto los recursos natura les que se consideran estra tégicos en el proceso de acumula ción como aquellosmenosalterados que corresponden a los que se ponen a salvaguarda (Comas-d'Argemir, 1998, p. 121). No obstante, la a rrolladora imagen del progreso, sustenta da en la oposición sociedad naturaleza, cada veztoman másfuerza los contra disc ursos. Éstos, a unque no son nuevosy han sido marginalesfrente a una práctic a «depredadora», siempre han estado presentes de manera «substantivista»frente a la economía formalista o de la maximiza ción del benefic io. Las intencionesy a cciones relaciona das por la protec ción y/o conservación de la natura leza, y que hoy se encamina a una nueva categorización de la natura leza y nuevos contenidos producto del cambio climátic 0 , son parte también de la empresa «civilizatoria occidental» (Brosius, Tsing y Zemer, 2005), representando una «nueva amenidad»y una «necesidad de la vida modema» (G uha, 1997).

Como empresa civiliza toria, el contra disc urso conservacionista puede ser a precia do tempranamente en visionarios (polític os y filántropos) que previeron valores inca lc ulables en la conserva ción de grandes bosquesy entre comunida desque tienen un marca do ac ento en el a utoc onsumo. En este sentido, desde hace varios sig los existe una disposic ión romántica hacia la naturaleza virgen y salvaje, favoreciendo una construcción histórica ambiental que corre en paralelo a la figura del extractivismo, agudizado dentro de la neoliberación de la Naturaleza. Por ejemplo, sabemos que, si bien la maderación del bosque resultó emblemática en téminos de intercambio comercial, es decir, tuvo valor monetario y desplegó importantescontingentes de fuerza de trabajo, identificando a regionesa tra vés de este recurso, ta mbién se desa rrolla ron procesos de vigilancia, regula ción y control con fines recreativospara poblacionesurbanas. En Europa hubo prohibiciones, nomas, procedimientos y una serie de regla menta ciones que configura ron polític as bastantes precisas en to mo a la apropiación y explotación de los recursos natura les (Bevila cqua, 1993; Diegues, 1996), que después fueron introducidasen otraslatitudes, lasque no dejan de serambiguasdesde la perspectiva del romantic ismo implicado, en la medida que hubo gustos diletantes que favorecieron la reinvención de regionesy definición de áreasespecialesa tra vés del excursionismo y tema lismo, estableciendo exc lusividad por clases adineradas. Mientras que, en Esta dos Unidos de Nortea méric a se suele identific ar protección ambiental con protec ción de la naturaleza salvaje (Guha, 1997).

De tal suerte que, la conservación del bosque y su entomo ha tenido ambigüedades y disonancias que se han movido entre la protección ambiental, la vida salvaje y formas de extra ctivismo. Observamos en la nomativa regula toria una expansión sistemática y planificada del valor de cambio de los recursos maderables, lo que tenía por objetivo también garantizar el abastecimiento requerido para la exportación y la demanda de la ciudad (Bevila cqua, 1993). Poseer en abundancia el recurso forestal no sólo significaba la posibilidad de satisfac ernec esida desdoméstic as básicas, ya que la ma dera fue funda mental para el desa rrollo y consolida ción de las ciudades antes del uso del hormigón, sino que además permitía garantizar la energía para la flota marina con fines comerciales, militares y de expansión. De tal manera que el recurso forestal maderable tuvo una importante connotación económica y política, por lo que su legislación en materia de usos y conservación, 
además, de necesaria, estaba legitimada.

Las tempranas nomativas sobre los límites de uso del bosque fueron forjando una visión clásica de la conserva ción como tierrasvaciadas de hombres y mujeres, estableciendo un «sistema de medidas sociales, socioeconómicas y téc nico- productivas rea lizadas dentro de los límites de un Estado o a escala intemacional» (Del Risco, 1982), las que estaban orientadas al buen uso de los recursos naturales, y la definición de áreas de absoluta restric ción. Se trataba de «conservar los recursos naturales, [lo que] no significa dejar de utilizarlos, sino ordenar su utilización juic iosa, de modo que no se agoten y que se aprovechen de manera perfectamente racional y efic iente»(Del Risc 0, 1982, p. 169). De este modo, con la regulación se despliega una nomenclatura relativa al ordenamiento, racionalidad y eficiencia del uso de los recursos naturales, siendo una forma de opera ciona lizar e instituc iona lizar el concepto de conservación, que de pa so usurpaba recursos a sociedades especializa das y privaba a las mismas del conocimiento de un «universo biótico»(Guha, 1997).

La conservación, a nte todo, establec e objetivosque pemiten ordenaren el espacio y en el tiempo las posibilidades de uso de recursos de un área, generando una zonificación que permite adecuartipos de uso y a ctividades que ayuden de manera ordenada a alcanzar las metas de protección, tanto desde lo biótico como desde lo escénico. Ferrero, indica sobre este proceso ubicado las disc usiones globales:

«a Unión Intemacional para la Conservación de la Naturaleza y los Recursos Naturales (UICN) ha sistematiza do la ca tegońa de áreas protegida scomo «una superficie de tierra y/o mar especialmente consagrada a la protección y al mantenimiento de la diversidad biológic a, así como de los recursos natura les y los recursos culturales asociados, y manejada a través de medios juńdicos u otros medios efic aces» (Loc. cit. Ferrero et al., 2013, p. 101).

En estos términos, la figura del Parque Nacional, como la describe Ramachandra Guha, se realza: «provee una oportunidad de calma, contraste, contemplación y afirmación de valorespara aquellosque viven la mayorparte de sus vida sen el mundo del trabajo»(1997, p. 38).

Conforme a lo señalado, hay una patrimonialización que produce una zonificación que contiene lugares que pueden ser catalogadas como intangibles, condic ionando percepciones y signific a dos, en cuanto son luga res que están a bsoluta mente al ma rgen de intromisión a ntrópic a, y otros, que son regula dos, y que pueden ser más a biertos ha cia ac tividadescomo el turismo y la educación a mbiental. Así, el proceso de protección patrimonial se inscribe en losprocesos de construc c ión polític a de lasregiones, tendientesa una definición de la ocupación del espacio que favorece una cierta imagen de la nación, de la mano de intereses ec onómic os, polític os y administra tivos. Así, poderes desiguales, gubemamentales, empresariales, ONGs, comunidades científic as y pobladores loca les definen las formas de oc upar, usar y conferir sentido al ambiente y al teritorio. 
Disc utir sobre este proceso, que siempre es sobre una imagen del futuro, es decir, sobre el sociosistema de la vida, tiene una historia de siglos. Sin embargo, mantiene vigencia superlativa, ya que en la actualidad se vuelve ingente producto de losca mbios climáticos, el aumento de la temperatura, la disminución de la biodiversidad, así como la necesidad de captura de carbono y la producción limpia; siendo una discusión necesaria en relación a las áreas protegidas, el uso de los recursos y las formas de vinculación y dependencia entre comunida des tra dic iona les y espec ia liza das, ya que como sostiene Guha (1994), cruza deterioro ambiental, escasez de recursos (o mala distribución de los mismos) y conflicto social.

Como conflic to, creemos aparece instituc ionalmente en una lectura simplifica da el fantasma de Garret Hardin (1968) y su célebre artículo «The Tragedy of the Commons». Éste, conc ibe a miembros de sociedadesque tienen bienes de usufructo común, con una incapacidad para tener restric ciones y poner límites a sus a c ciones so bre los ec osistemas. Cuestión que impedińa establecercuánto es lo suficiente que cada uno de losmiembrospuede extra er en términos energéticos. Esta figura desarrollada por Hardin, y que proviene de zonasque poseen como especia liza ción el ga na do y el uso de áreasde pastoreo, fa voreceńa una presión ambiental fuera de control, que expresańa en la llamada «tragedia de los comunes». Representando aquello que se vuelve contra la propia población y contrario al bien común y un objetivo de preservación ambiental (op.cit).

En este sentido, el modelo clásico de conservación se anticipa con este prejuicio sobre los bienes comunes, y ante la posibilidad de «a ruina» de los comunes. De modo que, las salidas económicas y ambientales frente a lo que podría ser tan ilimitado como un extra c tivismo ganadero y forestal, teminan siendo regulados con la privatización o la participación del Estado en el área identificada de valor, para asegurar la regulación, la sustentabilidad y sostenibilidad de los serviciosambientales. Así, el acceso a las tiemas bajo resguardo debe limitar el uso de sus recursos, por una incapacidad de los involucrados en contenerel alcance de susacciones, lo que pone al centro la disc usión las a uténtic asposibilidades del desa rrollo local y el bienestar de la población.

Para construir esta disc usión, que omite que los comunes ta mbién inc luyen y excluyen a sus miembros, y que es una sobre bienes protegidosque se transforman en comunes (nacionales y globales) porlas urgencias planetarias, tomamos el caso del Parque Nacional Cofre de Perote, Estado de Veracruz, uno de los más antiguos de México. Para expresar cómo la a utoridad, tratando de ser eficiente y cumplir con objetivos en estas escalas, instala una dicotomía falsa entre naturaleza segregada y población ejidataria, en la medida que no logra leer etnográficamente los procesos de codependencia de los campesinos con los recursos natura les y la posibilidad de comunalizar bienes que durante un siglo han esta do en el imaginario local como expresión de su identidad. De manera que, los intereses entre actorespugnan entre el ima ginario del futuro y la necesidad presente de contarcon recursos para la reproducción.

Ante la nec esidad de conservar luga res ec osistémic amente valiosos, hoy podemosencon- 
trar dos fómulas que podrían operar como tipos ideales: a) las que impiden todo uso y acción a ntrópica, y, b) las que pemiten un uso sustenta ble de los rec ursos disponibles para comunidades tradicionales. Éstas, representan por una parte, la comiente ambientalista clásica, que sustenta la línea de conservación y presenvación de la diversidad sin la intervención o presencia humana, y dónde las visitas serían privativas de comunida des científicasy con fines de investiga ción; y, por otra, una que podría mos lla ma r soc ioa mbienta lista, donde existe una transferencia de responsabilidad ética y ambiental a comunidades tradic iona lesa senta das, aspiránd ose a una rela ción de ca rá c ter equilibra do y a rmónic o, ya que pueden ser corresponsables de la conservación intergeneracional, la que se acercańa a una suerte de etnoconservacionismo.

En esta segunda perspectiva, las Áreas Nacionales Protegidas se complejizan en cuanto son sistema sque podemos considerarabiertos, ya que una cuestión es la mic rooga niza ción local, es decir, quienes viven dentro de los límites del área de conservación, y otra es la organiza ción sistémic a circundante a las áreas, y de las cuáles en muchas ocasiones dependen las poblacioneslocales. Es decir, poruna parte, existen rela cionescomerciales que implican captura, uso y transformación de recursos, lo que Guha (1994) llama lucha entre gente de losec osistemas y los omnívoros; y, por otra, rela c iones de c lientelismo, patema lismo, volunta des, liderazgos, fa voresy negociaciones con burócratas que se rea liza n con el extenor, pero que afectan el interior.

En estos términos, inicialmente, la cuestión es la relación con el Estado a través de sus funcionarios; o, cómo la autoridad promueve y socializa valores trascendentales y cata loga interés por lugares que destacan por su biodiversidad o cuestiones estéticas. Luego, cómo, directa o indirectamente, y con qué intensidad entra el mercado dentro de una lógic a formalista y/o extractivista verde. Sin embargo, la presentación en código binario resulta estrecho por dos cuestiones esenciales: 1) porque la declaratoria de un área de conservación, esa posteriori del uso que le han dado a los recursos poblaciones tra dicionales residentes, lasque han insc rito lasmarcas de su vida económica, reproductiva, a mbiental, simbólica y experiencia en el área protegida ${ }^{1}$; 2) porque el ca pitalismo ta mbién se legitima a tra vés de disc urso sconserva c ionista, especia Imente en su fa se neoliberal, en cua nto también existe un capitalismo verde.

En este sentido, en losorígenes de losparquesnacionalesenc ontramosuna connotación pragmática de movimiento de fronteras y de fortalecimiento del colonialismo intemo. La expulsión de laspoblacionesy losdec retosde expropiación, fueron elementoscomunespa ra hacerva ler lo que podría ser el temprano «derecho ambiental». A través del Estado, las áreas naturales protegidas se constituyeron como una forma particular de presencia institucional, definiendo, delimitando y visualizando estos temitorios en el ima gina rio nacional (Ferrero et al., 2013, p. 85).

\footnotetext{
${ }^{1}$ El caso norteamericano es uno de los más emblemáticos a éste respecto. Es importante mencionar que las tierras declaradas parques nacionales y que pasarín a «resguardo» gubemamental eran tierras habitadas, en el caso de Yellowstone por tribus Crow y Shosone quienes «abandonaron voluntariamente» la región o fueron expulsados por el ejército (Kuppe, 1999, p. 95-96).
} 
Este tipo de política s recibieron fuertes críticas durante el siglo XX, y en la súltimas déca das se planteó de forma sistemátic a la incorporación de otras va riables másallá de laspolíticoeconómicas y estético-naturales. En la actualidad, frente a las propias contradicciones u oxímorom que implic a que un área natural llena de ecosistemas y de cadenas trófic as sea puesta dentro de ciertos límites, se habla en términos más amplios de corredores biológicos y ecorregiones, para demostra que existen aspec tos móviles de la sespecies que implican características también binacionales como ocurre en el sur austral de Chile y Argentina, especialmente entre aquellos que profesan la ecología profunda (Rodríguez Torrent, Reyes y Mandujano, 2016), así como de la incomoración en la toma de decisiones de las poblaciones a sentadas dentro de las áreas natura les protegidas (ANP) y en áreas de influencia, bajo esquemas de gobemanza y justicia ambiental.

Hasta aquí, lo expuesto lleva en una primera línea a preguntas permanentes en tomo a la conserva ción, lasque pueden ser sintetiza das de la siguiente manera: ¿qué se debe conservar? ¿quiénes deben conservar? ¿con qué fines? ¿para quiénes? Luego, en una segunda línea, se debe interrogar también sobre el conocimiento generado en las ocupaciones preexistentes a la declaratoria: ¿a quién le pertenece? Laspreguntas, por una parte, siempre ponen el futuro al frente, poroposición a un pasa do que incomoda o genera cuotas de malestar, por otra, abre una importante discusión sobre el estatuto de los bienes comunes preservados.

\section{Parques Nacionales y áreas de consenvación en México}

Para el caso de México, losa ntecedentesjuńd ic osdel ma nejo de áreasde conserva ción, se registra n con el establec imiento de la Ley General del Equilibrio Ecológico en 1988, la cual indica en sus aspectos medula res las disposiciones para la preservación y restauración del equilibrio ecológico y la protección al ambiente; teniendo por objeto propiciar: el desarollo y aprovechamiento sustentable, establecer las bases para garantizar a toda persona a vivir en un medio ambiente sano, la definición de una política a mbiental y los instrumentos para su aplicación, así como garantizar la participación corresponsable de las personas, de forma individual y/ o colectiva (Congreso de los Estados Unidos Mexicanos, 2018).

El interés por conservar la vida silvestre fue una práctica arraigada en el mundo colonial, desde 1530, con la protec ción delemblemátic o bosque de Chapultepec (De la Maza, 1998). Luego, en 1917, con Venustiano Carranza se decretó la primera área natural protegida, El desierto de los Leones; con Lázaro Cárdenas, se muy rápidamente se decretaron 41 áreas de protección la gran mayońa Parques Nacionales (Gallegos, 2002). En paralelo, en casi todos los casos registra dos durante el siglo XX, las prác tic as de conserva ción a mbiental se orientaron hacia cuestiones forestales (Boyer y Orensanz, 2007), a postando por el control estatal en áreas donde había poca presencia del Estado. La justificación del posicionamiento del Estado definió una concepción evolucionista, estableciéndose un modelo que va porfuera de la vida local, ya que se consideraba que los campesinos carecían de la 
capacidad para administrar sus recursos, ya que dentro de su «negligencia primitiva» se comprometía el uso racional de los recursos forestales.

En estos términos binarios civiliza ción y barbarie, a nalíticamente la política de conservación tuvo c inco ejes: 1) consistió en tra tar de estimulary dotar de cierta conciencia y sensibiliza ción a los campesinos a tra vés de capacitaciones y propuestas de cooperativas de trabajo, con la idea de modemizar el pensamiento campesino; 2) una sanción sobre la transgresión de las nomativas, es decir, puso el ac ento sobre las prohibiciones; 3) sometera los campesinos al imperio de la legisla ción contribuye a su conversión en ciuda danos de una nación, porque se produce una integración simbólica y material en lo que puede ser evaluado como un proceso de violencia estatal; 4) generar un proceso de identidad a través de áreas y monumentos natura les en los lugares donde la autoridad era débil; y, 5) generar id entida des a mbienta les representa tivas del país. De modo que, se genera una temprana polaridad entre pensamiento local, la autoridad y el conocimiento c ientífico, y entre natura leza y justic ia so cial, obvia ndo los procesos bidirec ciona les y dialécticos entre sociedad y naturaleza, ya que el trabajo localmente realizado de gestión del bosque es algo más que una actividad económic a extractiva. Las prohibiciones inhibían lo que llamaríamos la figura antropológica de la totalidad mausseana, en cuanto a través del trabajo sobre estasáreas de conservación y de bienes comunes se están creando y recreando permanentemente las interacciones sociales, porque las relac iones materia les modific adas implic an la creación de universos simbólic os singulares, lo que sostiene a una comunidad desde la perspectiva de las interacciones (Wolf, 1987). Con ello, la hegemonía conservacionista impuso el conservadurismo científico frente a lo lógicas campesinas de uso controlado, vinculadas a una «filosofía de minimización del riesgo»(R. Guha y Gadgil, 1993, p. 95), el que operó «como una brocha con la que [se] borraban las variaciones locales en uso de teritorio, conocimiento ambiental y estructura social» (Boyer y Orensanz, 2007, p. 94); invisibilizando la identidad gestada entre los habitantes y su entorno, ya que las práctic as ejercidas por los productores y recolec tores loc a les sobre el universo biótic o son físicas, psic ológ ic as, simbólic as y materiales, con a plic aciones económicas, sociales, recreativas, estéticas y trascendentales.

Conforme a esto, la lógic a hegemónic a de loscientífic osdebe ser registrada ta mbién como «conflic to intermodal» (Guha y Gagdil, 1993, p. 95), ya que el modo de utilización de los recursos entra en contradicción con otro modo organizado de principios. O, «socioambiental», ya que refiere a «enguajes de valoración», diferenciados por hábitat, paisaje y merca do (Martínez Alier, 2011, p. 52-57) y esc enarios y procesos soc iales con una marcada polarización de intereses con relación al acceso, manejo, uso y a propiación de los recursos na tura les, así como a una confronta ción de visiones del mundo y percepciones culturales que pueden ser opuestas (Guzmán y Madrigal, 2012, p. 99-132). Entonces, si la existencia humana es una producción social colectiva, en este caso la primacía de la lógica científica se impone por sobre las condiciones adaptativas de las poblaciones, lo que no significa que laspoblaciones avecindadasoperen bajo una racionalidad cuyo objetivo 
sea el mercado y la maximiza ción de la ga nancia (verGodelier, 1976, 1989), sino que están produciendo también valores sociales comunes.

\section{E Parque Nacional Cofre de Perote}

El primer acercamiento con las localidades y poblacionales asentadas dentro del ANP, en el municipio de Perote, ubicado en la ladera poniente de la Montaña llamada Cofre de Perote, fue en elmarco del proyec to «Regiones so cioec onómica sfrente al ca mbio climático: transformaciones en los mercados de trabajo y tensiones socio-ambientales en un Área Natural Protegida en el esta do de Veracruz». Dadas las condic iones de violencia e inseguridad que prevalecen en la zona, el primercontac to fue con lasautoridadeslocales. Ahíse expuso el interés por conocer y recuperar cómo las poblaciones enfrentan el cambio c limático, así como registrar los cambios en el merca do de trabajo y reconocer la existencia (o no) de tensionesy conflic tos socioa mbienta lesen el Pa rque Nacional Cofre de Perote (PNCP). La respuesta fue favorable por parte de las autorida des munic ipales, las autoridades locales (agentes municipales y comisariado ejidal) y la población en general.

En el PNCP es común la presencia de académic os y orga nizaciones no gubemamentales orientadas a la conservación, principalmente la población tiene contacto con biólogos, ecólogose ingenieros forestales y en menor medida con académic os del área social.

En los primeros meses de trabajo de campo el espacio de contacto fue la asamblea ejidal (de a hí que el mayornúmero de entrevista dossean va rones). Ahí se log ró establecer rapport con autoridades y produc tores agríc olas. Este espacio también posibilitó ubic ara las mujeresorga niza das del Comité de Mujeres Unida spara la Conserva ción de losBosquesdel ejido El Conejo, ${ }^{2}$ así como contactar a otras mujeres interesadas en la toma de decisiones de la comunidad.

El trabajo de campo incluyó entrevistas, recomidos formales y caminatas en el bosque y parcelas de cultivo con los ejida tarios. También, largas c harlas con las mujeres en las cocinas, y no menos importante resultó la recolección de hongos, leña y hierbascon los niñosy jóvenes. Esto permitió registrary reflexionar sobre el uso de los recursosy las formas diversas de habitarel ANP, o lo que Guha y Gagdil (1993) lla man «modos de utilización de los recursos». Es decir, los contextos naturales en los que se asientan, responden y transforman los actores ${ }^{3}$.

El caso del PNCP o Nauhcampatépetl, decretado en 1937, corresponde a una de lasprimeras resenas natura les México. Abarca parte de los municipios de Perote, Xico, Ixhuacán y Ayahualulco, en la zona central del estado de Veracruz, ocupando poco más de 11,500

\footnotetext{
2 Sobre el origen y evolución del Comité ver Pineda, Sánchez, Vázquez, Lara y Ortega (2016).

${ }^{3}$ Guha y Gagdil (1993, p. 51) plantean que «el modo de utilización de los recursos» enriquece la comprensión de la esfera de producción a cuestiones como la flora, la fauna, el agua y los minerales, lo que identifica una ideología y un tipo de impacto ecológico particular, complementario a la idea de modo de producción.
} 
has. Como en otras ANP, la declaratoria de Parque Nacional (PN) se llevó a cabo en temitoriosoc upa dosparcia Imente porcomunida descon derechosa grarios, cuya dotación ejidal se concretó entre 1934 y 1936, es decir, previo al decreto.

La oc upación contemporánea del área está documentada desde la presencia de las haciendas (Hoffmann, 1989; J uárez Martínez, 1990; Ramos, Magnon, Piña, O 'Quinn y Cárdenas, 1984). La ha cienda, a finales del siglo XVII, como sistema de tenencia controlaba prác ticamente el $85 \%$ de la propiedad en la zona, con la presencia de tres haciendas: San J osé de los Molinos, Ximonco y Tenextepec. En estas propieda des se controlaba casi el 70\% de la riqueza forestal del Cofre de Perote, mientras el resto del bosque lo usufruc tuaban los naturales de la comunidad indígena de Santiago Ayahualulco. La población mayoritaria se a glutinaba alrededorde la cabecera munic ipal y de las grandeshaciendas. Posteriomente, con el reparto agrario producto de la revolución y el cambio en términos de tenencia de la tierra, se refomularon las relaciones de trabajo, propiciando un giro de lo forestal a lo a gríc ola y pecuario. Aunque se continuó con la explota ción forestal, principalmente con el desmonte para abrir paso a las parcelas agrícolas, poco a poco se fue dando mayor importancia a la agricultura, sobre todo porque las ganancias generadas por la venta de madera eran mínimas, los rendimientos quedaban en manos de los intermediarios constituidos por élites ma dereras que durante décadas explota ron sin límite el bosque, pese al decreto de ANP. En este sentido, las primeras cuatro décadas del decreto fueron letra muerta.

En la década de los setenta del siglo pasado se registró una intervención esta tal importante y un proceso de concientización de los habitantes sobre la conservación y la finitud de los recursos forestales ma derables y no maderables. Esta etapa estuvo marcada por una fuerte restricción al desmonte, se limitó el pastoreo con una tendencia a la desaparición, así como una limitación al uso de madera para la construcción o reparación de sus viviendas. Lo que transformó de manera radical la actividad económica de la población, experimentándose un abandono casi total de la actividad forestal, a la par que se incentivaba la actividad agńcola. Quienes no lograron adaptarse a este fuerte cambio tuvieron que migrar, para buscar otras ocupaciones que les pemitiera la sobrevivencia:

«Recuerdo que en esasfec hasse fueron fa milias completas, por lo menos unas treinta de esta comunidad, algunas pocas regresaron, porque dejaron a los abuelos, pero de otras nunca más supimos. Fue difícil, como comunidad, ver que tus parientes, a migos, se fueran del lugar que los vio nacer. Pero así fue» (Manuel, El Conejo, 2019).

En la actualidad, la principal actividad es la agricultura, ya sea dentro o fuera de la comunidad, en la partes de mayor altura se cultiva papa, y conforme la cota desciende, se pueden a preciar-en menormedida-cultivos de haba, maíz, frijol, cebada y ajo. Casi en su totalidad los cultivos son de temporada, de manera que la incertidumbre de la cosecha siempre está presente. A pesar de las dific ultades económicas para sostener los cultivos, estos son importantes para la reproducción de las familias, ya que la producción es principalmente para el autoconsumo. En el caso de la papa, un cultivo más comercial, una parte es oc upada para el consumo y otra es comercia liza da, ya sea en la cabecera muni- 
cipal o en ciudades aledañas, donde el precio no está asegurado, ya que puede variar dependiendo de la demanda y oferta, pero nunca pasa del medio dólarpor kilo. También hay presencia de ganado ovicaprino sin rebasar las 30 cabezas por unidad familiar. Algunos de los a nima les son cria dos para el a utoc onsumo, principa Imente en fiesta sfa milia reso festejos del pueblo. El resto de los a nimales son puestos a la venta y comprados por personas que suben a la montaña a «acaparar»el ganado menor.

En este contexto, se a precia que lascondic iones materia lesen lasque sobreviven lospobladores del PNCP se caracterizan por un reducido sistema de redes y oportunidades sociales (trabajo) y económicas (monoproducción), de manera que se encuentran permanentemente arrastrados a prácticas de mayor explotación de sus recursos naturales para su sobrevivencia. En la actualidad, el punto central de estas prácticas tendientes a la sobrevivencia, es que nos encontramos frente a un disc urso dominante que simultáneamente exalta «o eco»o «o verde»(producción verde, consumo verde, capitalismo verde, etc.) y pide transferencia de recursos y productos hacia las urbes, lo que no es necesariamente compatible con las posibilidades a mbientales y reproductivas de los habitantes de ésta área protegida.

A partir del acercamiento etnográfico, que demuestra un uso contenido y no expansivo del área, es importante resaltar que uno de los problemas históric os que establece la población a través de las entrevistas, es el escaso diálogo sobre el parque como bien común entre quienes operan el ANP y lo pobladores en relación a usos sustentables. Poco se sabe de los beneficios y de la importancia regional en téminos económicos y ambientales que tiene el PNCP a nivel localy regional, produciénd ose una permanente desvinc ulación entre quieneshabitan y func ionariosque «controla n», lo que tiene variasa rista ssobre la gobemanza del área. Cuestión que llamaremos desencuentro narrativo, ya que se trata de diferentes lugares de enunciación sobre lo que se entiende y prioriza en la conservación desde las instituc iones gubemamentales, lo que se traduce en tensiones que en la práctica se manifiestan al momento de ejecutar medidas concretas de conservación.

\section{Señala Armando:}

«Sabíamosque se nombró el parque nacional, pero mucho después de lo que decía el papel, ya con el decreto nosotros seguíamos usando el bosque, porque esa era nuestra forma de vida, nuestra manera de trabajar. Sí se cortaba el trozó (de ma dera) y se vendía, se pastaba el ganado, sí es verdad, porque vivíamos del bosque (...) pero ya mucho después se vio que esto no estaba bien, nos estábamosacabando el bosque, pero era la forma de vida (...) llegó la veda y ahí sí se a cabó, hubo casosen que te llevaban preso» (Ejido de los Pescados, 2017).J uan, completa la misma idea: «Mi frustración es tener algo que no podemosusar, es mío porque ahí está el papel, pero no es mío porque no puedo hacer nada. Sabesque si te metes con el bosque vasa la cárcel. Yo quisiera que nos dijeran qué podemoshacerpara beneficiamos. Sí sabemos que hay que cuidar y así lo hemos hecho por muchos años, pero deben entender que nosotros necesitamos vivir de algo » (Rancho Nuevo, 2017).

Nicandra señala lo siguiente: 
«Nos dicen que hay que cuidar, nos han traído cocina de barro para cortar menos leña, pero esas no son para acá, necesitamos de metal para que nos caliente en época de frío (...) no podemos no cortar leña, la ocupamos para cocinar, para calentar el agua para el baño, para sobrevivir en tiempo de frío. ¿Porqué no podemos cortar si está en terrenos nuestros? Nosotros cuidamos, sabemos que cortar qué no, que recogerque no. Nosotras sabemosque del bosque tenemospara vivin» (El Conejo, 2018).

Los registros obtenidos van siempre en la misma dirección. La opinión de los pobladores se inc lina por un bloqueo de la información que lesbrindan las instituciones gubemamentales que tienen injerencia en el manejo de la ANP, como es la Comisión Nacional de Áreas Protegidas y la Comisión Forestal. Principa Imente, son las sanciones sobre el uso del bosque másque losbenefic ioslo que prevalece en el disc urso; predomina un «no se puede», que «es la respuesta de siempre».

Otro poblador, señala:

«a última platica que tuvimos con CONANP fue para pedir apoyo para continuar con el pago por serviciosambientales, pero pues que ya no nos tocó. Nos dicen que es importante la conservación, que mantengamos esto, está bien, pero nosotros cuidamos y ¿a nosotros quién?»(Pedro, El Conejo, 2017).

En todos los casos, el bloqueo tiene un componente ideológico clásic o: áreas que deben estaral margen de toda intervención antrópica. Y, una negación de posibilidad de avanzara una transición productiva distinta, del tipo a groecológica. Nadie discute el papel de las ANP como sitios de conserva ción importantes en la historia ambiental de México, pero el sa crific io lo realizan los campesinos ejidatarios, ya que el uso del bosque es considera do daño, lo que está directamente asociado a la posibilidad de generación de humedad para lluvia, y provisión de a gua fuera del parque. Instituc iona Imente se ha sincera do menos esta variable entre los campesinos, y se ha sido menosflexible para reconocer los derec hos de propiedad. Estableciéndose su valor como un caballito de batalla dentro de losmecanismos para afrontar el cambio climático, ya que, por la posición geográfica del país, sus cond ic iones climáticas, orográfic as e hidrológicas, se encuentra como una de las zonas de importante vulnerabilidad climática (Aragonés, 2015; Betancourt Posada et al., 2014; Delgado, Cometta y Diaz, 2014; Kreft, Eckstein y Melc hior, 2016), lo que ha relevado su valor como área protegida.

Las Áreas Naturales Protegidas 4 , dentro de la Estrategia de Cambio Climático, protegen ecosistemas de alto valor, que contribuyen a reducir la vulnerabilidad ante el cambio climátic o y mitigar las emisiones de dióxido de carbono. Estableciéndoseles como uno de los grandes sumideros de carbono, siendo posible crear pautas para mejorarla calidad de vida de la población rural y urbana (CONANP, 2015). Fómula, de fortalecimiento y bienes-

\footnotetext{
${ }^{4}$ Actualmente la CONANP administra 182 áreas naturales de carácter federal que representan 90,839,521.55 hectáreas y a poya 388 Áreas Destinadas Voluntariamente a la Conservación, con una superficie de 417,562.27 hectáreas (CONANP, 2019).
} 
tar de las economías y poblaciones loc a les y regionales, entendida como la solución natural a nte el ca mbio c limá tico. De a cuerd o con la CONANP, (2015), los ecosistemas, en a monía con las comunida des huma nas, constituyen una de las herra mienta smá sefec tivas pa ra la conservación, permitiendo la adaptación de la biodiversidad y enfrentar los efectos del cambio climático, mediante la ampliación de corredores naturales que favorecen que las especies se adapten y ajusten sus áreas de distribución frente a las nuevas condiciones climáticas, incrementando su capacidad adaptativa.

El desarrollo sustentable se convierte en eje de la operación de programas de manejo de las ANP. De modo que la conservación a mbiental va en estrecha relación con la superación de la pobreza y margina ción de las comunidades asentadas en las ANP. En el caso particular del Parque Nacional Cofre de Perote, se puede constatar que la situación de pobreza y vulnerabilidad de sus habitantes es un hecho que no ha cambiado, aun con la emisión de su Programa de Manejo. De a cuerdo a los da tosproporcionadosporla CONAPO, lasloca lidadesubic adas dentro del ANP correspondientes al munic ipio de Perote se ubican en grado de margina ción alto (CONAPO, 2010). Esto signific a que el índice de marginación, que es un parámetro estadístico que coadyuva a la identifica ción de sec tores del país que ca recen de oportunida des para su desa rollo y de la capacidad para encontra rlaso generarlas, obliga a pensar en cómo las poblaciones sobreviven con el peso estructural que significa estar en un ANP5.

En este sentido, la determinación de dec retar una ANP en sus distintas modalidades ${ }^{6}$ ha implic ado el establecimiento de límites geográfic os bien definidos, los que no corresponden cartográfic amente a los usos tra diciona les de tipo material, simbólico y ambiental de las poblaciones, como se ha establecido en la antropología económica (i.e., el clásico trabajo sobre los Nuer de Evans-Pritchard). Una definición de límites administrativa o política sobre el teritorio, no representa los usos consuetudinarios sobre el espacio y cómo ha sido a propiado y transformado en lugar, lo téc nico cartografiado se contrapone al uso y signific a do del espac io. Quién designa los espa cios susc eptibles de conservación, y qué se puede hacer y qué no en las ANP, quién puede hacer uso de los recursos y bajo cuáles esquemas, debe contemplar altemativas para la moratoria de uso de los distintos recursos usa d os intergenera ciona Imente. Estas preguntas son extrema da mente importa ntes ya que la ANP como bien superior para preservarbienes na ciona lescomunes, implic a en un primer momento el establecimiento de parámetros para designarla como un núcleo teritorial conforme a un lenguaje de la valoración. No se reconoce su historia vinculada con el uso simbólic o y material de los recursos natura les, la cultura de trabajo liga da con los mismos y los derechos legales asocia dos a la asignación de tierras ta mbién quedan subordinados.

Lo a nterior refleja que la «elección»y designación de lugares sujetos a protección, ha sido una práctica unilateral, jerárquica y sin reconocimiento de sus habitantes. Y, más reciente-

\footnotetext{
${ }^{5}$ Se establecen cuatro dimensiones: educación, vivienda, ingresos y tamaño de localidad.

${ }^{6}$ Para el caso de México son resenvas de la Biosfera, Parques Nacionales, Monumentos Naturales, Áreas de Protección de Recursos Naturales, Áreas de protección de Fauna y Flora y Santuarios.
} 
mente, en los procesos de ampliaciones de las ANP, se han producido conflictos ante la poca participación de los habitantes en la generación del marco de acción para el uso de los rec ursosy a c tivida des ec onómic a sen las ANP (Merino y Hemá ndez, 2004; Za mora, 2017). Estas poblaciones rura lesque han mantenido un estrec ho vínc ulo con su a mbiente, para su reproducción biológica y cultural, en este caso relacionada con el bosque, se han tenido que ajustar a un esquema restric tivo mayor, que en sus primeras décadas fue totalmente inc ompatible con sus estrategias y práctic as de sobrevivencia, a saber la silvicultura y gana deńa. Por lo mismo, es importante mencionar que después de una fuerte restructura ción productiva (de forestal a agńc ola), los habitantes continúan vinculándose con el bosque ahora más en términos identitarios que productivos, a unque continúan desarrollando actividades de explotación como la recolección de leña, fundamental para la reproducción del grupo doméstico, así como la obtención de agua de manantia lesy a lgunosotros recursos no maderables.

Sin embargo, existe un velo en la infomación acerca del manejo del ANP. La que además de serpoco clara y a sequible para el lenguaje de los pobladores, presenta una contraposición de lenguajes de conocimiento, lo que ha genera do ciertas contiendas dentro y por el ANP. Por una parte, desde las instituc iones gubemamentales la conservación del bosque está porencima de lasnecesida desbá sic as de sobrevivencia de los pobladores, presentando una idea dicotómica (que parecía superada) entre ser humano-naturaleza; y por otra parte, el «deseo»de volver al estado «natural» de las cosas, sin hombres y mujeres. Asimismo, se mantiene una visión prejuiciada, en donde los campesinos siguen siendo los principales depredadores del bosque, ya que carecen de la capacidad (técnica e intelectual) para conservar sin intermediación extema, ya sea de instituciones públicas o privadas. En esta línea señala un investigador.

«Hace unas décadas atrás sí se requirió esfuerzo en el Cofre para reforestar porque estaba muy talado, pero hoy, ya no necesitas más, el Cofre se cuida solito, incluso no necesitas de la gente, la natura leza siguió su curso, necesitas vigilar eso sí, pero con gente o sin ella el Cofre se va a mantenen» (Investigador INEC OL, Xalapa, 2017).

Otro, indic a lo siguiente:

«Se ha hecho un gran esfuerzo para que los pobladores comprendan la importancia de conservar, noshemos va lido de universidadesy ONG spara que se den talleres y se haga producción científic a en elPN, por la gran riqueza en términos de biodiversidad y también se ha hecho un gran esfuerzo en la reforestación, involucrando a la poblac ión, pero es difícil porque ellos ven con otra lógic a las cosas, ellos quieren seguir su modo de vida anterior y eso no es compatible con la consenvación» (Funcionario CONAFOR, 2017).

En suma, las narrativa sentre los habita ntes y quienes ven «desde afuera » y tienen influencia sobre el manejo de las ANP, se muestran posturas no convergentes que dan cuenta de la polaridad entre el pensamiento local y el habitar, del Estado y sus funcionarios, y el del interés privado en tomo a la conservación. Observarla naturaleza como algo ajeno y establecer vigilancia sin facilitar el habitar para mantener el equilibrio, dejan ver cómo se van 
definiendo los espacios y los límites, el uso y manejo de los recursos que se han convertido en bienes comunes dentro del Estado federado. No sólo se ponen en juego las propias prácticas de sobrevivencia, sino se trastocan aspectos profundos de ca rácter simbólic o e identitario, como señalan Ovando y Ramos (2016), ya que produce nuevos antagonismos en tomo a la resignificación de sus temitorios, los que responden a distintas formas de invasión de sus espacios intersubjetivos de la cotidianidad.

\section{Resultados}

Las áreas de conservación han ganado notoriedad en las últimas décadas, ya que la expansión de la economía de merca do ha genera do una presión superlativa sobre losrecursos natura les. Esto, deviene en impacto ambiental de considerablesmagnitudes, ya que la lógica del consumo lleva a que muchos recursos sean destruidos-consumidos a un nitmo superior a su capacidad de regeneración, especialmente de bosques tropicales, y de manera imposible en los grandes bosques. En el caso de México, en las ANP existe una fuerte dependencia de las poblaciones rurales sobre los recursos naturales, las cuales se encuentran - en buena parte- en condición de pobreza y requieren de ellos para su existencia cotidiana, y a veces son demasiado pobres para ser verdes o ecológicos. Como hemos seña la do mása miba, estas poblacionesse vinculan con lasac tua lesáreas de conserva ción por medio de la actividad agrícola y forestal, así como a través de prácticas «menos» visibles pero cotidianas como el uso de recursos no maderables entre los que destaca la recolección de hierbas curativas, hongos, leña, actividades de caza y pesca, la dotación de agua, y otra sactivida des que han practica do históric a mente. Las restric ciones que implica la designación de ANP los sitúa en una posición de mayor vulnerabilidad al limitar el acceso a estos recursos, por lo que no solo es un problema de sobrevivencia, sino también de memoria de vida. Por ello, la tensión que se observa es entre la orga niza ción del trabajo que permite la reproducción y el control porparte del Estado de losmedios de producción, ya que se están restringiendo los circ uitos de intercambio y la propia manifestación de sus percepciones y categorizaciones sobre la naturaleza y la vida.

Este escenario de cambio social es doble: 1) es un problema de economía política, en cuanto la decisión esun problema de poderque se concreta en la esfera del trabajo; 2) de ecología política, ya que condiciona el acceso a los recursos, y altera los procesos a daptativosy el mismo manejo de los recursos. De modo que la conserva ción corresponde a un proceso polític o y social (Alcom, 2005), en loscua lespartic ipa el Esta do y por el cual se ordenan los recursos natura les dentro de procesos ecológicos. Así, la exclusión de los ejidatarios del uso de los recursos dentro del concepto de ANP, es decir, de a quello que se convierte en «bienescomunes» 0 «bienesnacionales colec tivos» que son nec esa rio conservar porque se trata de ecosistemas significativos, pero sin usufructo material de ellos, no permite que naturaleza y cultura sean parte de una misma conceptualización, o visualizar las interacciones y la necesaria y recíproca relación. Esto, comporta un modelo de preservación más bien rígido, el que no logra visua liza r los usos, a para tos tec nológ ic os y disposi- 
tivos menta les tra diciona les en relación al entomo, así c omo el comporta miento partic ular asociado a los lugares de usufruc to.

Entonces, si se reconoce a la población ejidataria como un actorque introduce elementos de desequilibrio, no es difíc il entender la poca partic ipa ción que éstostienen en losprogramas de conserva ción o la oposición a estas polític as y programas, así como las divisiones y tensiones dentro de las localidades entre aquellos beneficiados por un detemina do programa de ayuda y los ignoradoso pejudicados por el mismo. Esto implica un reto que no siempre se considera: contemplar a los pobladores no sólo como prestadores de servicios ec osistémicos, sino como sujetos con nec esida des de reproduc c ión y consumo. De manera que, mientras no existan otras a ltemativas fuera o dentro del ANP que les proporcionen lo necesario (o mínimo) para su sobrevivencia, continuarán «trasgrediendo» los límites sobre uso de recursos natura les establecidos instituciona Imente como medidas de conservación. En estos términos, la trasgresión de las noma tivas sobre áreas protegida s es contradisc urso de apropiación y una modalidad de ejercicio de derechos políticos. Si bien los avances científic os permiten «c alc ular»y «definir»áreas de alto valor de conservación, por ejemplo en el caso del establecimiento de Regiones Terrestres Prionita rias en México (Arriaga et al., 2000), poco se considera el a porte en términos heuństic os y prác tic os del saber loc al, de su historia, del reconoc imiento de rela c iones so cioa mb ienta les preexistentes. Por ello, a demás de recuperar las narrativas propias de una relación permanente con los recursos teritoria Imente disponibles, se tiene que prestaratención hacia las práctic as y vínculos de lospobladores con el a mbiente y la dependencia. Esto esdecisorio en una mejor instrumentación y efica cia de polític as públicas de conservación, no solo en términos técnicos sino como un proyecto complejo, con identidad, arraigo y de reconocimiento de interdependencia entre los habitantes y los recursos naturales. Como plantea Durand «a conservación ambiental sólo será posible cuando (...) se logré construir una relación con lo natural que signifique y emocione a laspersona sy donde la na tura leza se trasfome en un verdadero componente de identidad»(2005, p. 215).

Frente a lo señalado, tanto en el diseño como en la ejecución de leyes y programas que identific an estos bienes locales que pasan a ser bienes comunes especiales en la esfera nacional, y que ta mb ién pasa n a serc omunesg lobalmente, esnec esa rio haceruna relectura de la interpreta ción de la rela ción serhuma no-natura leza, a sí c omo de la instituc ionalizac ión del concepto de conservación. Lo importante, es entenderqué y porqué se requiere conservary en qué téminos se puede «vivir» dentro de un ANP, y c uáles son la saltema tivas para que las poblaciones vivan con dignidad y con los recursos suficientes para alcanzar la reproducción social y biológica.

A partir de la experiencia en el Parque Nacional Cofre de Perote, podemos señalar que habita ren el ANP para estosc a mpesinos ha signific a do un reto en términos de sobrevivencia. El decreto es exclusionario y es altamente restric tivo para permitir la reproducción de la pequeña producción a grícola, ya que todoslos recursosnaturalesson propios de la existencia localizada por décadas y es intergeneracional. Y, la ejecución del mismo decreto a 
partir del Programa de Manejo, se ha llevado a cabo de manera «poco participativa», es decir, sin una cartografía que exha ustiva que sustente la rela ción entre c ultura y natura leza. Para los habitantes del munic ip io de Perote, conc retamente a quellas establecidas dentro del PNCP, el decreto implicó una reconversión productiva y una nueva forma de relacionarse con el bosque, cada vez más a jena y menos vinculante a sus prácticas. La existencia de un bosque que no puede «ser tocado» y de tierras que no pueden ser «utilizadas», ha sembrado sentimientos de frustración, enojo y suspicacia en tomo al ANP.

La reconversión productiva implicó un cambio en la economía campesina y un quiebre en la cultura laboral. Del manejo forestal se pasó casi por completo a la apertura de pequeñas parcelas para sembrar papa en las partes altas y en menor medida maíz, haba y frijol en las partes bajas. Hasta la década de losochenta la papa fue un producto rentable. La crisis actual del tubérculo se atribuye a distintas causas: la baja demanda del producto como consecuencia de la crisis ec onómic a del país; el cambio en lospatrones de consumo que desplazó las papas de color o nativas cultivadas en el Cofre, por la papa alfha producida en los estados del norte del país y Michoacán; y, la falta de capital para obtener los insumos necesarios para el cultivo, a una do a los pocoso nulos a poyos gubema menta les.

La crisis de la papa y las restric ciones foresta les del ANP a cotaron la s altema tiva s labora les en la comunidad. Hoy en día se registra una mayor movilidad, principalmente de los varonesquienes se emplean en el trabajo agrícola fuera de la comunidad (en municipios aledaños o incluso en estados de la frontera Norte), y en fábricas de block (de hormigón) ubicadas al pie de la montaña.

Pa ra las mujeres, la soportunida des en la comunidad son aún más esc asas. Porlo que quién decide emplearse debe sa lirfuera de su loca lidad. Lasmujeresse desplazan hac ia la cabecera municipal o a Xalapa para realizar trabajo doméstic o o emplearse en el comercio. Tal es el caso de Manina, una mujer adulta que pasó más de 30 años como trabajadora del hogar.

«Trabajo en casa desde los 13 años, primero en Perote, después me llevaron a Xalapa (capital del estado). Aquí no había como trabajar, ganar dinero, el campo ya no daba ni para comer, asíque con permiso de mi papá me fui. Luego ya cuando venía al pueblo se a nimaban las muchachasa salir (...) coloqué a variasen casas, primero me pedían para el quehacer pero también coloqué para nanas o cuidadoras de viejitos en Xalapa (...) menos pesa do y pagaban bien» (Marina, El Conejo, 2018).

Las reducidas oportunida des de trabajo en las comunida des son un reflejo de las limitacionesmanejo y de uso de los recursos locales, siguiendo a Kuppe (1999, p. 102), la «participación»o integración de miembros de lascomunidades, o si se quiere el reconocimiento de poblaciones a vecindadas, es, en el mejor de los casos, limitada a la función como guarda parques o guías turísticos.

En esta función de tipo top-down, los habitantes se integran al nivel más bajo en la jerarquía administrativa de los equipos que protegen la vida silvestre; $y$, consecuentemente, sin 
ninguna oportunidad de tomar decisiones sustanciales de a lgo que es considera do a hora un bien común que se debe preservar, y antes un bien que se tenía en propiedad. Sin embargo, es importante menciona r que existen esfuerzos y práctic as cotid ia nas a nivel comunitario para la conservación del bosque por ejemplo, el mantenimiento de tinas ciegas ${ }^{7}$, la poda y limpia, y cuando se requiere la organización para combatir incendios. Estas actividadesson organiza dasen lasasambleasejidales, lascua les se llevan a cabo cada mes, por lo que son temas prioritarios.

Asimismo, se puede prever que su propia estructura sociopolític a asociada al ANP con sus funciones conservacionistas propias del a prendizaje intergeneracional, se verá sustituida por la sestruc turas jerá rquic as y buroc rátic as ca rac terístic as de la s instituc iones esta ta les.

Lo señalado, demuestra que la creación de unidades de conservación como las ANP, no pueden garantizar siempre el c umplimiento de los objetivos ambientales. En lo esencial, se necesita disponer de recursos materiales, financieros y humanos para que la conservación de estos bienes comunes pueda ser gestiona do y armoniza da en función de un equilibrio entre conservación y reproducción social y biológic a de las poblaciones. Mientras, las unidades campesinas sigan siend o vulnerables, los ejerc ic ios de c omuna liza ción seguirán siendo escasos. Se requiere de un permanente y progresivo involucramiento entre los sectores que representan a la burocracia y la institucionalidad, y aquellos que han vivido en el área de conservación desde antes de su declaratoria.

\section{Consideraciones finales}

Las posibilidades de una comunaliza ción de los bienes y una transición de comunidades hacia un modelo protector de bienes comunes nacionales y globales, pasa por una voluntad de Estado porgenerar condiciones de reconocimiento de las poblaciones locales. La posibilidad que esto ocura, no puede depender de una definición de área protegida a tra vés de la ausencia de poblacioneso sin acción antrópica. La oposición entre «sociedad y parques >como sostiene Ferrero (2018), siguiendo el modelo norteamericano anclado en la noción de wildemess (vida natural o salvaje), tiene efec to destruc tores frente a lascomunidades que viven de recursos primarios, llegando a producir «refugiados ecológicos» en dirección a lasciudades tras oportunidades de empleo (Guha, 1994: 137). Sin embargo, el contexto de esta orientación esen rela ción a procesosind ustria lesy a vance de la sociedad urba na, es dec ir, «salvac ionista». La extra polación es ina propia da sobre grupos especia lizados que se corresponden al perfil de la autoreproducción, y no puede serpensada al margen de la historia adaptativa de las poblaciones humanas.

Es difíc il sostener el modelo dominante sin considerar las historias productivas. Precisamen-

\footnotetext{
7 También nombradas zanjas trincheras son excavaciones que se realizan en curvas a nivel de 40 centímetros de ancho x 40 centímetros de profundidad y 2 metros de longitud se utilizan para incrementar la infiltración del agua de lluvia al subsuelo, disminuye la erosión al frenar los escumimientos superficiales.
} 
te, el éxito de ser eficiente en el cuidado, preservación y manejo a mbiental de áreas ricas en biodiversidad, está en el reconocimiento y la integración de las poblacionales locales; evaluando sus condiciones de vida, la dependencia de algunos recursos y sus prácticas reproductivas. Es difíc il a rgumenta rque las práctic as de comunidadesa socia dasal Parque Nacional Cofre de Perote, sean menos efic ientes que lo que han sido las de otros partic ulares y empresas privadas que explotaron el bosque, o el mismo Estado. Si se enfrenta una crisis sistémica y global, y se argumenta a favor de la conservación, elevando las Áreas Nacionales Protegidasa la categoría de «bienes comunes na cionales», y extensivamente a «bienescomunesglobales», al menosse debe intentarla restitución de derechospara fortalecer intereses comunes y a reinventar la historia a comunidadescodependientes, para la administración de los territorios especiales. Una respuesta necesaria a desafíos conservacionistas, está en que las comunidades sean capaces de apropiarse o generar nuevos significa dos (Escobar, 1996) en vista de objetivos que compa tibilic en sus necesidades con imperativos globales.

\section{Referencias}

Alcom, J. (2005). Dances around the Fire: Conservation Organizations and Community-Based Natural Resource Management. En Communities and Conservation: Histories and Politics of Community Based Natural Resource Management (p. 37-68) Walnut Creek, CA: Alta Mira Press.

Aragonés, A. M. (2015). Consec uencias del cambio climátic o sobre la migra ción: Un a nálisis para México. Ciudad de México, México: Universidad Nacional Autónoma de México, Instituto de Investigaciones Económicas.

Arraga, L., Espinoza, J. M., Aguilar, C., Martínez, E., Gómez, L., \& Loa, E. (2000). Regiones terrestres prionitarias de México. Ciudad de México, México: Comisión Nacional para el Conocimiento y Uso de la Biodiversidad.

Ba rkin, D., \& Lemus, B. (2015). Soluc iones Locales para la J ustic ia Ambiental. En F. De Castro, B. Hogenboom, \& M. Baud, Gobemanza Ambiental en América Latina (pp. 297-330).Buenos Aires, Argentina: CLACSO.

Baud, M., De Castro, F., \& Hogenboom, B. (2011). Environmental govemance in Latin America: Towards an integrative research agenda. European Review of Latin Americ an and Caribbean Studies, 90, 79-88.

Betancourt Posa da, A., Campos Ortiz, G. T., Cruz Mańn, J. E., Fritsche, K., J eg litzka, E., Loyd, M., Pérez Galvis, A. B., Riva Pala cios de lcaza, E., And rée Roy, M., \& Stric kland, V. (2014). ¿Cuánto vale la sabiduńa tradicional? El papel asignado a los conocimientos de los pueblos originarios en El Capital Natural de México. En Del Monologo a la Polifonía. Proyectos supranacionales y saberes indígenas en la gestión de áreas naturales protegidas (1990-2010) (pp. 82121). Ciudad de Méxic o, México: Universidad Nacional Autónoma de México, Facultad de Filosofía y Letras, Consejo Nacional de Ciencia y Tecnología.

Bevila cqua, P. (1993). Las políticas a mbientales: ¿qué pasado? Algunas reflexiones. Historia y Ecología, 147-169.

Boyer, C. R., \& Orensanz, L. (Eds.). (2007). Revolución y patemalismo ecológico: Miguel Ángel de Quevedo y la política forestal en Méxic o, 1926-1940. Historia Mexic ana, 57, 91-138.

Brosius, P., Tsing, A., \& Zemer, C. (Eds.). (2005). Communities and Conservation: Histories and Politics of Community-Based Natural Resource Management. Alta mira Press.

Comas-d'Argemir, D. (1998). Antropología Económica. Barcelona, España: Ariel.

CONANP. (2015). Estrategia de Cambio Climático desde las Áreas Naturales Protegidas: Una Convocatoria para la Resiliencia de México (2015-2020). Ciu- 
dad de México, México: Secretańa de Medio Ambiente y Recursos Naturales.

CONANP. (2019). Comisión Na cional de Áreas Natura les Protegidas. Recuperado de https://www.gob.mx/conanp/que-hacemos

CONAPO. (2010). Grado de margina ción por localidad para el Municipio de Perote. Grado de marginación por localidad. Munic ipio de Perote. Recuperado de http:// www.mic ro rregio nes.gob.mx/catloc/LocdeMun. a spx? tip o $=$ c la ve\&c a mpo $=10 c \&$ ent $=30 \&$ mun $=128$

Congreso de los Estados Unidos Mexicanos. (2018). Ley General de Equilibrio Ecológico y la Protec ción al Ambiente.

De la Maza, R. (1998). Una historia de las Áreas Naturales Protegidas en México. Natura Mexicana. Áreas naturales protegidas, 31-48.

Del Risco, E. (1982). La conserva ción y los jardines botánic os. Revista del J ardín Botánico Nacional, 3(1), 167-195.

Delgado, G. C., Cometta, A., \& Díaz, B. F. (2014). Cambio climátic o global, transformación agraria y soberanía alimentaria en América Latina. Buenos Aires, Argentina: CLACSO.

Diegues, C. (1996). O mito modemo da natureza intocada. Quito, Ecuador: Editora Huic itec-NUPAUB/USP.

Durand, L. (2005). Los mitos y la conservación a mbiental. Líder: revista labor interd isc iplinaria de desa rollo regional, 13, 215-226.

Esc obar, A. (1996). La invención del TercerMundo, Construc c ión y dec onstrucc ión del desa rollo. Popayán, Colombia: Editorial Noma.

Fa rinós Dasí, J . (2008). G obemanza teritorial para el desa rollo sostenible: Esta do de la cuestión y agenda. Boletín de la Asociación de Geógrafos Españoles, 46, 11-32.

Ferrero, B. G . (2018). Tras una definición de área protegida. Apuntes sobre la conservación de la naturaleza en Argentina. Revista Universitaria de Geografía, 27, 99-117.

Ferrero, B. G., Arizpe, N., \& Gómez, E. (2013). Definiendo la conserva ción: El ca so del Parque Nacional lguazú, Argentina. Ecología política, 46, 85-90.

Gallegos, C. (2002). Áreas naturales protegidas en México en el siglo XX. Ciudad de México, México: Instituto de Geografía, UNAM.

Godelier, M. (1976). Antropología y economía. ¿Es posible la antropología económica? En Antropología y Economía (pp. 279-333). Barcelona, España: Anagrama.

Godelier, M. (1989). Lo ideal y lo material. Pensamiento,, economías, sociedades. Madrid, España: Taunus.

Guha, R., \& Gadgil, M. (1993). Los hábitats en la historia de la humanidad. Ayer, $11,49-110$.

Guha, Ramachandra. (1994). El Ecologismo de los pobres. Ecología política, 8, 137-151.

Guha, Ramachandra. (1997). El a mbientalismo estadounidense y la preservación de la naturaleza: Una crítica tercemundista. Ecología política, 14, 3346.

Guzmán, M., \& Madrigal, D. (2012). Naturaleza y sociedad en San Luis Potosí: Apuntes para una ecología política. En L. Durand, F. Figueroa, \& M. Guzmán, La Naturaleza en contexto. Hacia una ecología política mexicana ( $p$ p. 95136). Méxic o: Universidad Nacional Autónoma de Méxic o, Centro de Investiga ciones Interd isc iplina rias en Ciencias y Huma nida des, Centro Regional de Investiga c iones Multid isc iplina ria s. leakey

Hardin, G. (1968). The Tragedy of the Commons. Science, 162(3859), 1243-1248.

Hoffmann, O. (1989). De los hacendados a los forestales: Manejo del espacio, dominación y explotación del bosque en la Sierra Madre Oriental (Cofre de Perote). Trace/ : Procesos Mexic anos y Centroameric anos, CEMCA - Centro de Estudios Mexicanos y Centroamericano, 31-49.

J uárez Martínez, A. (1990). Crónica de un ecocidio: El llano de Perote. Anuario VII, 55-75.

Kreft, S., Eckstein, D., \& Melchior, I. (2016). Global Climate Risk Index 2017. Who suffers most from extreme weather events? Weather-related loss events in 2015 and 1996 to 2015. Germanwatch e.V. Recuperado de https:// 
www.gemanwatch.org/12978

Kuppe, R. (1999). Indígenas y medio a mbiente: Conservacionismo a espaldas de los guardianes de la tierra. Boletín de Antropología Americana, 35, 95-104.

Lemos, M. C., \& Agrawal, A. (2006). Environmental Govemance. Annual Review of Environment and Resources, 31(1), 297-325.

Martínez Alier, J. (2011). El ecologismo de los pobres: Conflictos a mbientales y lenguajes de valoración. Barcelona, España: Ic aria Editorial.

Merino, L., \& Hemández, M. (2004). Destrucción de instituciones comunita rias y deterioro de los bosques en la Reserva de la Biosfera Mariposa Monarca, Michoacán, México. Revista Mexicana de Sociología, 66(2), 261-309.

Ovando, C., \& Ramos, R. (2016). Imaginarios geográficos en tomo a la franja fronteriza de Tarapacá: El estado y los habitantes/migrantes. Scripta Nova: revista electrónic a de geografía y ciencias sociales, 20(529), 1-25.

Pineda, M. del R., Sánchez, L. R., Vázquez, S., La ra, R., \& Ortega, R. (2016). Opciones de participación de género para la protección de bosques en el Parque Nacional Cofre de Perote, México: PFNM y podas (pp. 26-33). Veraccruz, Méxic o:Universidad Veracruzana.

Ramos, H., Magnon, C., Piña, J. M., O'Quinn, A., \& Cárdenas, R. (Eds.). (1984). La lucha campesina en Veracruz, Puebla y Tlaxcala. Ciudad de México, México: Ediciones Nueva Sociología.

Rodríguez Torrent, J. . C., Reyes Herrera, S., \& Mandujano Bustamante, F. (2016). Reconfiguración espacial y modelos de apropiación y uso del teritorio en la Patagonia chilena: Migración por cambio de estilo de vida, parques de conservación y economía de la experiencia. Revista de geografía Norte Grande, 64, 187-206.

Rosales Ortega, R., \& Brenner, L. (Eds.). (2015). Geografía de la gobemanza: Diná mic as multiescala res de los procesos económico-ambientales. México: Universidad Autónoma Metropolitana, Unidad Iztapalapa/ : Siglo Veintiuno Ed itores.

Wolf, E. (1987). Europa y los pueblos sin historia. Ciudad de México, Méxic o: FCE.

Zamora, C. (2017). Vivir en la Reserva: Las comunidades rurales, actores de la conservación. En L Ruiz- Montoya, G. Álvarez- Gordillo, N. Ramírez- Marcial, \& B. Cruz- Sa lazar, Vulnerabilida d social y biológica a nte el ca mbio climático en la Reserva de la Biosfera Selva El Ocote. San Cristóbal de Las Casas, Chiapas, México (pp. 67-86). Ciudad de México, México: El Colegio de la Frontera Sur. 